\title{
Charge Density Waves in Electron-Doped Molybdenum Disulfide
}

\author{
Mohammed K. Bin Subhan, Asif Suleman, Gareth Moore, Peter Phu, Moritz Hoesch, \\ Hidekazu Kurebayashi, Christopher A. Howard,* and Steven R. Schofield*
}

Cite This: Nano Lett. 2021, 21, 5516-5521

Read Online

ACCESS | 니 Metrics \& More | 回 Article Recommendations | st Supporting Information

ABSTRACT: We present the discovery of a charge density wave (CDW) ground state in heavily electron-doped molybdenum disulfide $\left(\mathrm{MoS}_{2}\right)$. This is the first observation of a CDW in any $d^{2}$ (column 6) transition metal dichalcogenide (TMD). The band structure of $\mathrm{MoS}_{2}$ is distinct from the $d^{0}$ and $d^{1}$ TMDs in which CDWs have been previously observed, facilitating new insight into CDW formation. We demonstrate a metal-insulator transition at $85 \mathrm{~K}$, a $25 \mathrm{meV}$ gap at the Fermi level, and two distinct CDW modulations, $(2 \sqrt{3} \times 2 \sqrt{3}) \mathrm{R} 30^{\circ}$ and $2 \times 2$, attributable to Fermi surface nesting (FSN) and electron-phonon coupling (EPC), respectively. This simultaneous exhibition of FSN and EPC CDW modulations is unique among observations of CDW ground states, and we discuss this in the context of band folding. Our

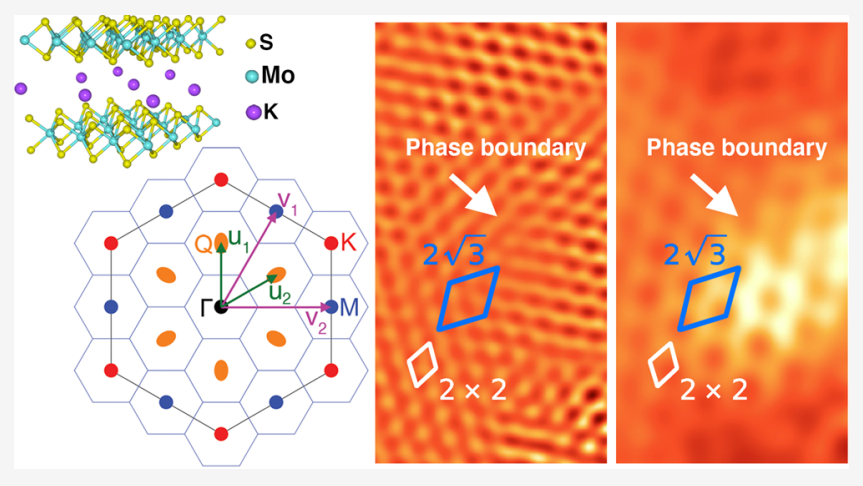
observations provide a route toward the resolution of controversies surrounding the origin of CDW modulations in TMDs. KEYWORDS: Charge density wave, metal-insulator transition, $\mathrm{MoS}_{2}$, intercalation, STM, tunneling spectroscopy

trongly anisotropic crystals that confine charge carriers to $\checkmark$ two dimensions exhibit a rich diversity of correlated ground states, including charge density waves (CDWs), spin density waves, and superconductivity. However, despite decades of intense effort, there are still large gaps in our understanding of the mechanisms underpinning the formation and competition between such states. New experimental observations of correlated states can provide litmus tests for competing theoretical models. CDWs are a periodic spatial oscillation of charge density, accompanied by a lattice distortion, that occur in crystalline materials due to electron-electron and electron-phonon interactions. ${ }^{1-3}$ Despite intense investigation, the physics of CDW formation remains a topic of vigorous debate, ${ }^{4,5}$ and the connection to other exotic electronic ground states, most notably superconductivity, remains controversial. ${ }^{6}$

Transition metal dichalcogenides (TMDs) are two-dimensional (2D) layered materials that are tailorable by varying the elemental composition, coordination, symmetry, layer number and separation, and doping. ${ }^{7,8}$ TMDs thus provide excellent opportunities to investigate fundamental condensed matter physics in reduced dimensions. CDWs have been discovered in the semimetallic column $4\left(d^{0}\right)$ TMDs, $\mathrm{TiSe}_{2}{ }^{9}$ and $\mathrm{TiTe}_{2}{ }^{10}$ and in the metallic column $5\left(d^{1}\right)$ TMDs $\mathrm{VS}_{2}{ }^{11} \mathrm{VSe}_{2}{ }^{12}$ $\mathrm{NbSe}_{2},{ }^{4,13} \mathrm{TaS}_{2}{ }^{14}$ and $\mathrm{TaSe}_{2}{ }^{15}$ CDWs have not been previously observed in column $6\left(d^{2}\right)$ TMDs, which are typically band semiconductors. However, density functional theory calculations have indicated the possibility of CDW formation in heavily doped bulk ${ }^{1,17}$ and monolayer $\mathrm{MoS}_{2},{ }^{18,19}$ and a recent study reported anomalies in the temperature dependence of the sheet resistance in electron-doped $\mathrm{MoS}_{2}$, suggesting the possibility of a CDW phase transition. ${ }^{20}$ There are two popular mechanisms for CDW formation in TMDs: Fermi surface nesting (FSN; the favored model in $\mathrm{VSe}_{2}, \mathrm{TaS}_{2}$, and $\mathrm{TaSe}_{2}{ }^{3,12}$ ), which requires coupling of the Fermi surface and leads to the opening of a small energy gap centered at the Fermi energy; and momentum-dependent electron-phonon coupling (EPC; favored in $\mathrm{NbSe}_{2}$ and $\mathrm{VS}_{2}{ }^{11}$ ), which can occur in the absence of strong connections within the Fermi surface. In many cases, not all aspects of the data are adequately described by either model.

$\mathrm{MoS}_{2}$ is a band semiconductor, column $6\left(d^{2}\right)$ TMD with a trigonal prismatic $(2 \mathrm{H})$ ground state (Figure $1 \mathrm{c}){ }^{7}$ The band edges derive from $4 d$ orbitals, $^{7}$ and the bulk material has a 1.29 $\mathrm{eV}$ indirect band gap ${ }^{21}$ (Figure 1a). In reciprocal space, the valence band maximum is at the zone center $(\Gamma)$, while the conduction band minimum is located midway along $\Gamma-\mathrm{K}$, producing a 6-fold degenerate conduction band with electron pockets at the points labeled $\mathrm{Q}$ in Figure $1 \mathrm{a}, \mathrm{b}$. With electron doping, $\mathrm{MoS}_{2}$ undergoes a metal-insulator transition at a free

Received: February 17, 2021

Revised: June 30, 2021

Published: July 6, 2021 
(a)

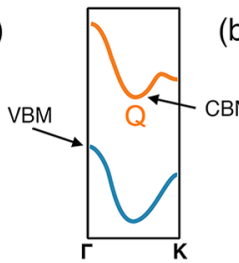

(c)

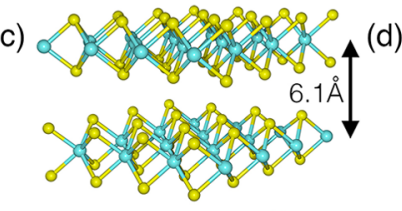

(e)

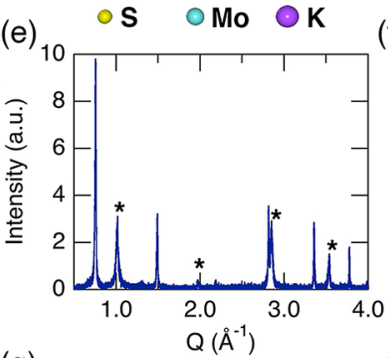

(g)

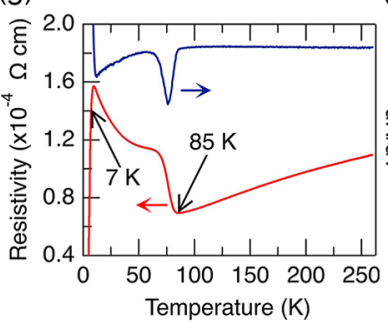

(b)

(d)
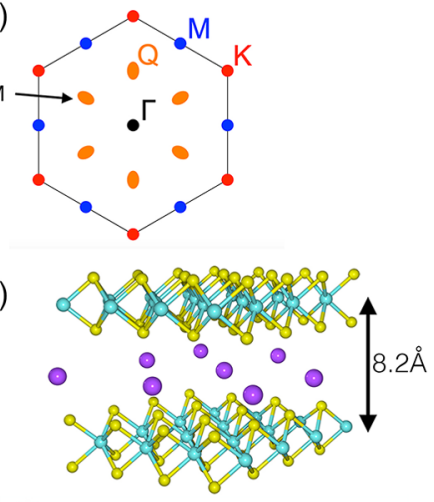

(f) $\widehat{\text { o }}$

(h)

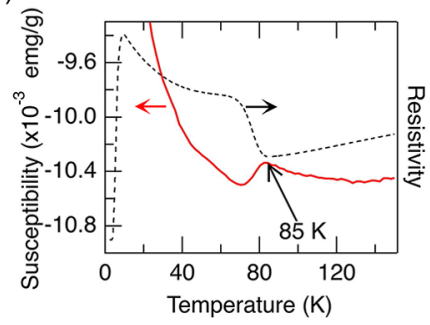

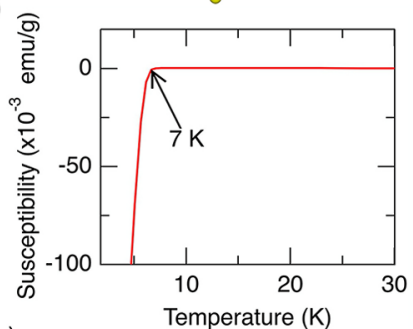

Figure 1. (a) $\mathrm{MoS}_{2}$ band structure schematic highlighting valence/ conduction band maximum/minimum (VBM/CBM) (adapted from ref 27). (b) Brillouin zone with high-symmetry points $\Gamma, \mathrm{K}, \mathrm{M}$, and low-symmetry point Q. (c) Crystal structure of $\mathrm{MoS}_{2}$ and (d) of $\mathrm{K}_{0.4} \mathrm{MoS}_{2}$. (e) $\mathrm{K}_{0.4} \mathrm{MoS}_{2} \mathrm{X}$-ray diffraction; asterisks denote unintercalated $\mathrm{MoS}_{2}$ peaks. (f) Magnetic susceptibility (10 Oersted). (g) Resistivity (red trace) showing metal-insulator (85 K) and superconducting transitions $(7 \mathrm{~K}) ; \mathrm{d} R_{S} / \mathrm{d} T$ is shown on the right axis (blue trace). (h) Magnetic susceptibility (red trace) with a large applied field $\left(10^{4} \mathrm{Oe}\right)$ with resistivity data overlaid (dashed curve; right axis).

carrier density of $n_{2 \mathrm{D}}=6.7 \times 10^{12} \mathrm{~cm}^{-2}$ and exhibits a sharp onset of superconductivity at $6.8 \times 10^{13} \mathrm{~cm}^{-2}$. 22

Here, we present the discovery of a CDW ground state in bulk potassium-intercalated (and therefore electron-doped) $\mathrm{MoS}_{2}$ with a simultaneous exhibition of FSN and EPC derived modulations. We demonstrate a metal-insulator transition at $85 \mathrm{~K},(2 \sqrt{3} \times 2 \sqrt{ } 3) \mathrm{R} 30^{\circ}$ and $2 \times 2 \mathrm{CDW}$ modulations via atomic-resolution scanning tunneling microscopy (STM) and a $25 \mathrm{meV}$ energy gap at the Fermi level via tunneling spectroscopy. The $2 \sqrt{3}$ modulation is perfectly matched by a nesting vector connecting the conduction band pockets, while the $2 \times 2$ modulation matches a theoretically predicted phonon-mode softening at the $\mathrm{M}$ point. ${ }^{18}$ We discuss that the two modulations are simple linear combinations of one another, suggesting that the driving mechanisms may be coupled via band folding.

Potassium ions were intercalated into the van der Waals gaps of a bulk $\mathrm{MoS}_{2}$ sample using the well-established lowtemperature liquid ammonia method. ${ }^{23}$ Briefly, high-quality $\mathrm{MoS}_{2}$ crystals (Manchester Nanomaterials) were degassed $\left(523 \mathrm{~K},<10^{-6} \mathrm{mbar}\right)$ then combined with potassium dissolved in liquid ammonia at $218 \mathrm{~K}$. Potassium intercalation (Figure 1d) completed after $\sim 24 \mathrm{~h}$. X-ray diffraction (XRD) was measured in a reflection geometry (Philips X'Pert) on cleaved samples in an airtight beryllium dome. Magnetic susceptibility measurements (Quantum Design MPMS-7) were performed on samples held in a plastic capsule and sample straw. Fourterminal contacts were attached to the sample using Epotek H21D silver epoxy and transport measurements (Keithley 2400 SMU and Stanford Research Systems SR830) were made on a coldfinger below $10^{-5} \mathrm{mbar}$ with a $1 \mathrm{~mA}$ current and 10 $\mathrm{Kh}^{-1}$ heating rate. STM measurements (Omicron LT-STM) were performed on samples cleaved under ultrahigh vacuum $\left(<5 \times 10^{-10} \mathrm{mbar}\right)$ at room temperature to produce an atomically clean surface of a bulk intercalated sample, and then cooled to $5.5 \mathrm{~K}$ for STM measurement.

XRD confirms the crystalline quality of our samples (Figure 1e); the $00 l$ out-of-plane peaks are shifted with respect to their unintercalated positions, demonstrating the expected $2.2 \pm 0.1$ $\AA$ increase in the layer separation. ${ }^{23}$ Magnetic susceptibility and four-terminal resistivity measurements (Figure if and g) confirm the onset of superconductivity at $T_{\mathrm{c}}=7.0 \pm 0.5 \mathrm{~K}^{23,24}$ The room temperature resistivity $\left(R \sim 1 \times 10^{-4} \Omega \mathrm{cm}\right)$ decreases linearly with temperature (Figure $1 \mathrm{~g}$, red trace), as expected. ${ }^{25}$ At $85 \mathrm{~K}$, we find a pronounced step increase of $\sim 5$ $\times 10^{-5} \Omega \mathrm{cm}$, marking the location of a metal-insulator transition. Also at $85 \mathrm{~K}$ we find an abrupt decrease $(-0.02$ $\mathrm{emu} / \mathrm{g}$ ) in magnetic susceptibility (Figure $1 \mathrm{~h}$ ). These features are characteristic of the opening of an energy gap at the Fermi level, and similar behavior has been attributed to CDW transitions in $\mathrm{VS}_{2}{ }^{11}$ and $\mathrm{TaS}_{2}{ }^{26}$

Figure 2a displays a topographic STM image of a region free from step edges or adatoms, and that is characteristic of images acquired using different samples and tips. The Fourier transform of this image (Figure $2 \mathrm{~b}$ ) exhibits hexagonal spots corresponding to a lattice constant, $a=3.14 \pm 0.07 \AA$, in agreement with the calculated lattice constant, $3.176 \AA$, ${ }^{28}$ confirming that we are imaging the surface sulfur atoms of the cleaved sample. A 0.2 monolayer surface coverage of potassium ions might be expected if half of the potassium ions in the cleaved layer remain on the surface after cleaving. However, we do not observe any surface potassium ions in our images and attribute this to the surface potassium ions diffusing to step edges or elsewhere while the sample is at room temperature before it is loaded into the STM and cooled. Several defects in Figure 2a appear as protrusions several nanometers in diameter superimposed on the surface atomic lattice. Similar defects, attributed to molybdenum vacancies or antisites, have been shown to locally enhance the $\mathrm{MoS}_{2}$ interlayer coupling 29,30

Figure $2 \mathrm{c}$ shows a higher resolution image acquired with a lower imaging bias magnitude $(-100 \mathrm{mV})$. This image and the corresponding Fourier transform (Figure 2d) reveal a longerranged periodicity of $5.8 \pm 0.2 \AA$, i.e., $2 \pm 0.2$ times the $1 \times 1$ lattice, which we interpret as a $2 \times 2 \mathrm{CDW}$ modulation. We notice that the apparent intensity of the $2 \times 2$ modulation varies within the image (Figure $2 \mathrm{c}$ ), suggesting a nearly commensurate CDW phase, as observed in STM images of $\mathrm{NbSe}_{2}{ }^{13}$ and copper-intercalated $\mathrm{TiSe}_{2}{ }^{31}$ Examining again the Fourier transform of the larger area image in Figure 2b, we see the $2 \times 2$ spots are also faintly visible in this data. Indeed, in all images of the $\mathrm{K}_{0.4} \mathrm{MoS}_{2}$ surface acquired with sufficient resolution we find evidence for a $2 \times 2$ periodicity to a greater or lesser extent depending on the imaging parameters 
(a)

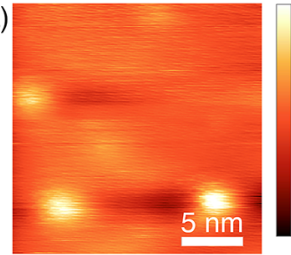

(c)

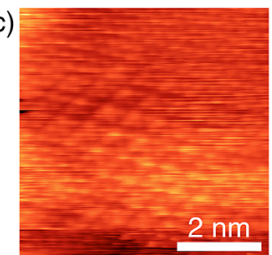

(e)

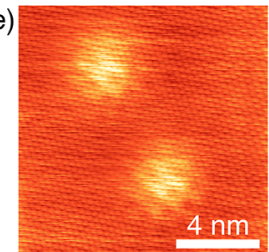

(g)

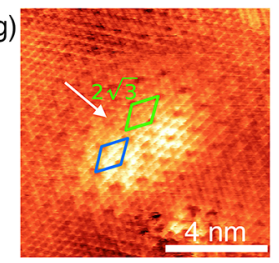

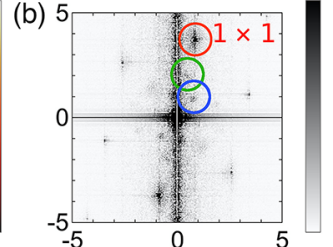

(d)

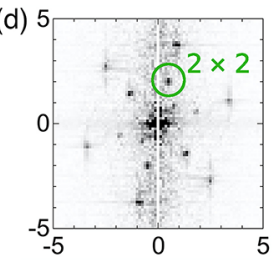

(f)

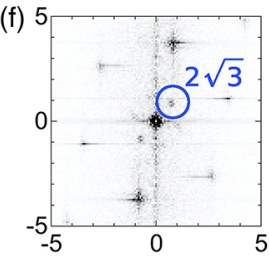

(h) 5

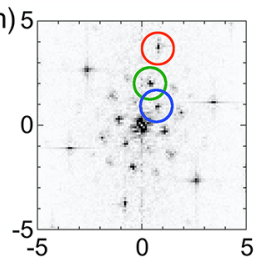

Figure 2. (a,c,e) STM topographic images of $\mathrm{K}_{0.4} \mathrm{MoS}_{2}(5.5 \mathrm{~K})$ and $(\mathrm{b}, \mathrm{d}, \mathrm{f})$ their corresponding $2 \mathrm{D}$ Fourier transforms. Bragg spots corresponding to the $1 \times 1$ surface sulfur lattice are seen in all images, while to a lesser or greater extent $2 \times 2$ and $2 \sqrt{3}$ periodicities are also observed (see text). Image parameters: (a) $-150 \mathrm{mV}, 20 \mathrm{pA}$, z-range $1.5 \mathrm{~nm} ;(\mathrm{c}, \mathrm{e})-100 \mathrm{mV}, 30 \mathrm{pA}$, z-range $700 \mathrm{pm}$. FFT axes are in $\mathrm{nm}^{-1}$ units. (g) Filled-state STM image of a pair of closely spaced defects in $\mathrm{K}_{0.4} \mathrm{MoS}_{2} .1 \times 1,2 \times 2$, and $2 \sqrt{3}$ periodicities can be seen in the image and its Fourier transform in panel (h). A phase slip boundary (white arrow) exists between the two regions of $2 \sqrt{3}$ periodicity, which we highlight on panel $(\mathrm{g})$ by adding a blue rhombus to indicate the phase of the $2 \sqrt{3}$ modulation at the bottom left defect site, and a green rhombus to indicate the $2 \sqrt{3}$ phase of the top right defect.

and location on the surface, suggesting that in $\mathrm{K}_{0.4} \mathrm{MoS}_{2}$ we have a nearly commensurate $2 \times 2 \mathrm{CDW}$ state present everywhere throughout the sample surface.

The image in Figure 2e shows an area where two defects are present. Here, we find an additional periodicity, distinct from both the $1 \times 1$ lattice, and the $2 \times 2$ modulations. The Fourier transform (Figure 2f) demonstrates that this periodicity is characterized by a $10.4 \pm 0.2 \AA$ real space vector, which is rotated $30^{\circ}$ with respect to the $1 \times 1$ lattice. We note that within uncertainties, this periodicity is $2 \sqrt{3}$ times the $1 \times 1$ unit cell vector $(=10.7 \pm 0.4)$, and we therefore label this as a $(2 \sqrt{3} \times 2 \sqrt{3}) \mathrm{R} 30^{\circ}$ modulation of the $\mathrm{K}_{0.4} \mathrm{MoS}_{2}$ surface. Unlike the $2 \times 2$ modulation, the $2 \sqrt{3}$ modulation is strongly enhanced within the vicinity of the defect sites. We found such $2 \sqrt{3}$ modulations always occur at such defects sites; e.g., the $2 \sqrt{ } 3$ spots are visible in Figure $2 b$ due to the defects in the corresponding STM image, and further examples are shown in Figures $2 \mathrm{~g}$,h and S1. Modulations of the local density of states in the vicinity of defects can occur due to quasiparticle interference (QPI); ${ }^{32}$ however, we rule out QPI in the present case since the modulation is dispersionless ${ }^{32}$ (see the spatially resolved tunneling spectroscopy presented below). It is possible also to draw comparison to $\mathrm{NbSe}_{2}$, where the $3 \times 3$ CDW modulation was found to persist above $T_{\mathrm{CDW}}$ in patches surrounding defects. ${ }^{13}$ However, our measurements of defects in $\mathrm{K}_{0.4} \mathrm{MoS}_{2}$ at 5.5, 10 , and $77 \mathrm{~K}$ show negligible change in the spatial extent of the $2 \sqrt{ } 3$ modulation surrounding the defects with temperature (see Supporting Information Figure S1). Moreover, we also observe phase slip boundaries in the $2 \sqrt{3}$ modulation for closely spaced defects, similar to observations of phase boundaries in CDW modulations seen in $\mathrm{NbSe}_{2}{ }^{33}$ and $\mathrm{TiSe}_{2}{ }^{34}$ We show an example of this in Figure $2 \mathrm{~g}$, which shows a filled-state STM image where two defects are present (the corresponding Fourier transform is shown in Figure $2 \mathrm{~h}$ ). The two defects exhibit $2 \sqrt{3}$ modulations, but these two modulations are not in phase, leading to a phase slip boundary between them, which we highlight with a white arrow in Figure $2 \mathrm{~g}$. Further analysis and discussion of this phase slip boundary can be found in Figure S2. The implication of these observations is that the $2 \sqrt{3}$ modulation can be attributed to a CDW whose enhancement at defect sites is an intrinsic property, and not due to QPI or an incompletely formed CDW phase.

Other non-CDW explanations for the observed $2 \times 2$ and $2 \sqrt{ } 3$ periodicities can be ruled out. The precise arrangement of the potassium ions in $\mathrm{K}_{0.4} \mathrm{MoS}_{2}$ is not known; ${ }^{23,28}$ however, the $\mathrm{K}_{0.4} \mathrm{MoS}_{2}$ stoichiometry ${ }^{23}$ and hexagonal symmetry of the crystal suggests a hexagonal arrangement of the average positions of the potassium ions, with a lattice constant of $(5 / 2)^{1 / 2} a$. Thus, even allowing for all possible rotational orientations of the potassium ion layer with respect to the top $\mathrm{MoS}_{2}$ layer, we can rule out both a direct influence of the potassium ion positions and Moire interference between the $\mathrm{MoS}_{2}$ and potassium ion layers as explanations for either periodicity. Moiré effects due to strain at defect sites can be additionally ruled out by the observation that the characteristic length of the modulation does not vary with distance from the defect center as would be expected for a point-source lattice distortion derived Moiré interference.

Figure 3 presents scanning tunneling spectroscopy (STS) measurements of $\mathrm{K}_{0.4} \mathrm{MoS}_{2}$. Point spectra show a bulk band gap of around $1.2 \mathrm{eV}$ (Figure 3a), consistent with previous measurements ${ }^{30}$ and calculations. ${ }^{28}$ Higher-resolution measurements at the conduction band edge (Figure $3 \mathrm{~b}$ ) show the band minimum is located $\sim 25 \mathrm{meV}$ below the Fermi level, in agreement with ARPES measurements of potassium-doped $\mathrm{MoS}_{2}{ }^{35}$ and a similar band shift in copper intercalated $\mathrm{TiSe}_{2} .{ }^{31}$

We also find an energy gap at the Fermi level (Figure $3 b, c$ ); we measure the gap as the point where a quadratic fit to the gap intersects the conduction band and take the gap to be twice this value, yielding $2 \Delta=24 \pm 4 \mathrm{meV}$. There was no measurable difference between spectra taken on or away-from defect sites, demonstrating that the gap occurs across the entire surface.

We have measured spatially resolved conductivity, $g(r, V)$, where $r$ is the tip position and $V$ is the sample bias (see Figure $\mathrm{S} 3$ ); when integrated over $r$ (Figure 3c), this yields good agreement with the point spectroscopy in Figure $3 \mathrm{~b}$. We deconvolve contributions associated with the different superlattice modulations by taking a 2D Fourier transform of $g(r, V)$ for each value of bias to obtain conductivity as a function of the reciprocal space vector $q$. We identify in this data the expected $1 \times 1,2 \times 2$, and $2 \sqrt{3}$ reciprocal lattice vectors and show the intensity variation of these spots as a function of bias in Figure $3 \mathrm{~d}$. The observation that these spots occur at the 

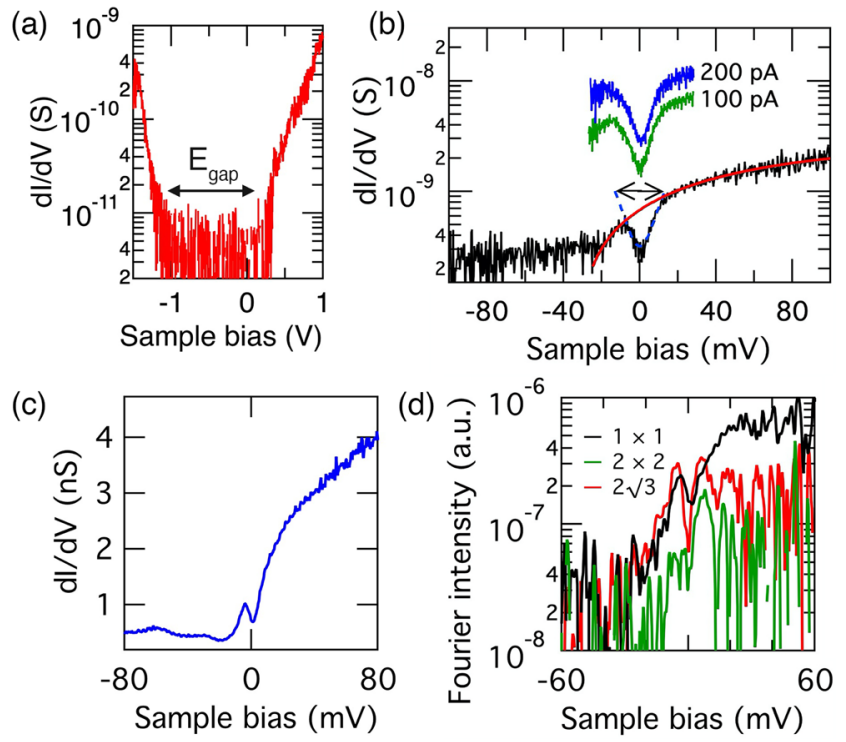

Figure 3. Point tunneling spectroscopy of $\mathrm{K}_{0.4} \mathrm{MoS}_{2}$ highlighting (a) the $\sim 1.2 \mathrm{eV}$ band gap and (b) the conduction band minimum at -25 $\mathrm{meV}$ and gap at the Fermi level; curve fits are to the band edge (red) and energy gap (dashed blue). (c) Spatial average of $\mathrm{d} I / \mathrm{d} V$. (d) Fourier spot intensities for the $1 \times 1,2 \times 2$, and $2 \sqrt{3}$ lattice spots (see Figure $\mathrm{S} 3$ for corresponding $\mathrm{d} I / \mathrm{d} V$ images at $\pm 5 \mathrm{mV}$ ). Tunneling spectra were acquired by measuring current as a function of voltage with the feedback loop off. Set-point parameters: (a) $-1.5 \mathrm{~V}, 50 \mathrm{pA}$; (b) black: $-100 \mathrm{meV}, 30 \mathrm{pA}$; green $-30 \mathrm{meV}, 100 \mathrm{pA}$; blue: -30 $\mathrm{meV}, 200 \mathrm{pA}$. All data $5.5 \mathrm{~K}$, except panel (b) $10 \mathrm{~K}$.

same values of $q$ for each value of bias (see Figure S3) rules out QPI origins for these modulations, and we do not observe any additional spots for any values of the applied bias. The $1 \times 1$ spot intensity variation (Figure $3 \mathrm{~d}$ ) closely matches the spatially averaged conductance (Figure $3 \mathrm{c}$ ). In contrast, the $2 \sqrt{3}$ spots exhibit a gap feature and peaked intensities either side of the Fermi level. The intensity variation of the $2 \times 2$ spots follow similarly to the $2 \sqrt{3}$ spots, although the signal-tonoise ratio is insufficient to say conclusively whether or not the gap exists in the $2 \times 2$ data. Concentrating on the $2 \sqrt{3}$ curve, which has the better signal-to-noise ratio, we measure peak maxima at $\pm 3.1 \pm 0.1 \mathrm{mV}$, i.e., separated about the Fermi level by a width of $6.2 \pm 0.2 \mathrm{mV}$. Alternatively, if we measure the width from the outer edges of the peaks, we find a width of 26 $\pm 2 \mathrm{mV}$. These widths compare well to the gap width estimation from our point spectra in Figure $3 \mathrm{~b}$. Thus, the spatially resolved tunneling spectroscopy data shown in Figure 3 , and the fact that the Fermi level gap in STS spectra occurs far from defect sites where the $2 \times 2$ modulation persists but the $2 \sqrt{ } 3$ modulation does not, suggest that the energy gap at the Fermi level correlates to both the $2 \times 2$ and $2 \sqrt{3}$ spatial modulations observed in our STM and STS images.

Our observations of a metal-insulator transition at $85 \mathrm{~K}$ and a $24 \pm 4 \mathrm{meV}$ energy gap centered at the Fermi level are suggestive of a FSN-driven $\mathrm{CDW}$ in $\mathrm{K}_{0.4} \mathrm{MoS}_{2}$. The necessary FSN vectors exist in the $\mathrm{K}_{0.4} \mathrm{MoS}_{2}$ electronic structure due to the fact that potassium intercalation of $\mathrm{MoS}_{2}$ creates electron pockets at the $\mathrm{Q}$ points. The length of the vector, $\mathbf{q}$ (Figure $4 \mathrm{a})$, that connects the Fermi surfaces at $Q$ is $|\boldsymbol{\Gamma}| / 2$, i.e., $|\mathbf{q}|=\frac{|\mathbf{b}|}{4} / \cos \frac{\pi}{6}=b / 2 \sqrt{3}$, where $\mathbf{b}$ is the reciprocal lattice vector. There are three pairs of such nesting vectors, each rotated $30^{\circ}$ to the $1 \times 1$ surface sulfur lattice. Thus, the vectors (a)

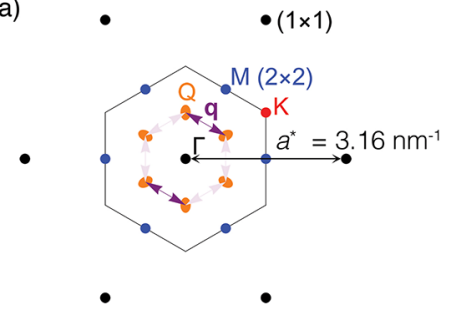

(b)

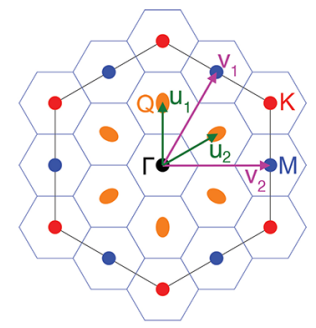

Figure 4. (a) Reciprocal lattice (black spots) and Brillouin zone with high symmetry points $\Gamma, \mathrm{K}$, and $\mathrm{M}$. Band minima at the low symmetry points, $\mathrm{Q}$ lying half way along $\Gamma-\mathrm{K}$, are shown. (b) Brillouin zone drawn with an overlaid $(2 \sqrt{3} \times 2 \sqrt{3}) \mathrm{R} 30^{\circ}$ unit cell. Vectors $u_{1}, u_{2}$ and $v_{1}, v_{2}$ indicate the $\mathrm{Q}$ and $\mathrm{M}$ points, respectively.

q connecting neighboring $Q$ points provide an excellent match to our observed $(2 \sqrt{3} \times 2 \sqrt{3}) \mathrm{R} 30^{\circ}$ modulation in STM and STS data. This provides strong evidence for a FSN-driven $2 \sqrt{3}$ CDW phase in $\mathrm{K}_{0.4} \mathrm{MoS}_{2}$ that is localized at or enhanced by defects in the $\mathrm{MoS}_{2}$ sheets. The reason for this enhancement at defects is that the defects locally enhance the interlayer coupling, ${ }^{29,30}$ resulting in a lowering of the band edge at the $Q$ point and thus an increased occupancy of the bulk-like electron pockets ${ }^{7}$ and a corresponding enhancement of the $2 \sqrt{3}$ FSN.

However, FSN cannot explain the appearance of the $2 \times 2$ periodicity in our STM data, because the electronic bands at the M-point are much higher in energy than the band minima.? However, calculations of the $\mathrm{MoS}_{2}$ phonon band structure as a function of electron doping have demonstrated phonon softening at the $\mathrm{M}$ point, becoming imaginary for electron doping levels exceeding $\sim 0.14$ electrons per molybdenum atom. $^{18,36}$ The $\mathrm{M}$ point lies on the Brillouin zone boundary along the reciprocal lattice vector direction (Figure 4a) and as such is inherently associated with a $2 \times 2$ periodicity. This strongly suggests that momentum-dependent EPC can account for the formation of the $2 \times 2$ phase in $\mathrm{K}_{0.4} \mathrm{MoS}_{2}$.

Thus, our observations of a metal-insulator transition at 85 $\mathrm{K}$, the opening of an $\sim 25 \mathrm{meV}$ energy gap at the Fermi level, and the real space observation of a $2 \sqrt{3}$ modulation in STM and STS data provide compelling evidence for the existence of a FSN-driven CDW phase, where the nesting vector connects electron pockets at the $\mathrm{Q}$ points. This $2 \sqrt{3}$ phase is locally enhanced by defects in the $\mathrm{MoS}_{2}$ sheets that locally alter the interlayer coupling and enhances the electron pockets at the $Q$ points. Simultaneously, we observe a nearly commensurate $2 \times$ 2 modulation that matches a predicted $2 \times 2$ EPC-driven $\mathrm{CDW}$ due to phonon softening at the $\mathrm{M}$ point. As has been a feature of several previous reports of CDW phases in TMDs, ${ }^{3,4}$ it is not possible to fully describe all of our data with either a FSN or EPC model independently.

We suggest that the coexistence of the $2 \times 2$ and $2 \sqrt{ } 3$ phases is possible in part due to the effect of band folding. We illustrate the reciprocal space vectors of the $2 \sqrt{3}$ periodicity $\left(u_{1}\right.$ and $\left.u_{2}\right)$ and $2 \times 2$ periodicity $\left(v_{1}\right.$ and $\left.v_{2}\right)$ in Figure $4 \mathrm{~b}$, and 
it can be seen that these are simple linear combinations of one another (e.g., $v_{1}=u_{1}+u_{2}$ ). Thus, band folding established by the shorter periodicity (illustrated by the hexagonal tiling in Figure $4 \mathrm{~b}$ ) will result in the band minimum at $\mathrm{Q}$ and the soft phonon mode at $\mathrm{M}$ being mapped back onto the zone center, providing an opportunity for the two mechanisms to couple. The fact that the two modulations are simple linear combinations of one another also provides an explanation for why the Fermi level gap we measure in STS spectra contains 2 $\times 2$ as well as $2 \sqrt{ } 3$ periodicity.

We have presented the discovery of a CDW ground state in $\mathrm{K}_{0.4} \mathrm{MoS}_{2}$, which is also the first observation of a CDW phase in a $d^{2}$ (column 6) TMD. Owing to the unique band structure of $\mathrm{K}_{0.4} \mathrm{MoS}_{2}$ compared to $d^{0}$ and $d^{1} \mathrm{TMDs}$, our results provide new insight into the formation mechanisms of CDWs. We observe a metal-insulator transition at $85 \mathrm{~K}$, an $\sim 25 \mathrm{meV}$ energy gap centered at the Fermi level, and $2 \times 2$ and $2 \sqrt{ } 3$ periodicities that can be explained, respectively, by EPC and FSN mechanisms. The $2 \sqrt{3}$ periodicity is observed exclusively near defects, suggesting that the coexistence of FSN and EPC phases can be attributed to their delicate sensitivity to the band edge and the strength of the interlayer electronic coupling. Moreover, our observations suggest that CDW phases might be discoverable in other column 6 TMDs such as $\mathrm{MoSe}_{2}, \mathrm{WS}_{2}$, and $\mathrm{WSe}_{2}{ }^{37}$ at high electron-doping and for which investigation might provide further insight into the physics of CDW formation.

\section{ASSOCIATED CONTENT}

\section{SI Supporting Information}

The Supporting Information is available free of charge at https://pubs.acs.org/doi/10.1021/acs.nanolett.1c00677.

STM images of $2 \sqrt{3}$ modulations at defect sites acquired at 5.5, 10, and $77 \mathrm{~K}$. STM images and analysis of phase slip boundaries. Spatially resolved tunneling spectroscopy of $2 \sqrt{ } 3$ modulation (PDF)

\section{AUTHOR INFORMATION}

\section{Corresponding Authors}

Christopher A. Howard - Department of Physics and Astronomy, University College London, WC1E 6BT London, United Kingdom; (1) orcid.org/0000-0003-2550-0012; Email: c.howard@ucl.ac.uk

Steven R. Schofield - Department of Physics and Astronomy, University College London, WC1E 6BT London, United Kingdom; London Centre for Nanotechnology, University College London, WC1H OAH London, United Kingdom; (1) orcid.org/0000-0002-0727-3015; Email: s.schofield@ ucl.ac.uk

\section{Authors}

Mohammed K. Bin Subhan - Department of Physics and Astronomy, University College London, WC1E 6BT London, United Kingdom

Asif Suleman - Department of Physics and Astronomy, University College London, WC1E 6BT London, United Kingdom; London Centre for Nanotechnology, University College London, WC1H OAH London, United Kingdom

Gareth Moore - Department of Physics and Astronomy, University College London, WC1E 6BT London, United Kingdom; London Centre for Nanotechnology, University College London, WC1H OAH London, United Kingdom
Peter Phu - Department of Physics and Astronomy, University College London, WC1E 6BT London, United Kingdom

Moritz Hoesch - Photon Science, Deutsches ElektronenSynchrotron (DESY), 22607 Hamburg, Germany; (i) orcid.org/0000-0002-0114-2110

Hidekazu Kurebayashi - London Centre for Nanotechnology, University College London, WC1H OAH London, United Kingdom; Department of Electronic and Electrical Engineering, University College London, WC1E 6BT London, United Kingdom

Complete contact information is available at: https://pubs.acs.org/10.1021/acs.nanolett.1c00677

\section{Notes}

The authors declare no competing financial interest.

The data created during this research are openly available via zenodo.org at 10.5281 /zenodo.3696856.

\section{ACKNOWLEDGMENTS}

This research was financially supported by the Engineering \& Physical Sciences Research Council (EP/L002140/1).

\section{REFERENCES}

(1) Grüner, G. Density Waves in Solids; Perseus Publishing: Cambridge, MA, 1994; pp 1-85.

(2) Zhu, X.; Cao, Y.; Zhang, J.; Plummer, E. W.; Guo, J. Classification of charge density waves based on their nature. Proc. Natl. Acad. Sci. U. S. A. 2015, 112, 2367-2371.

(3) Rossnagel, K. On the origin of charge-density waves in select layered transition-metal dichalcogenides. J. Phys.: Condens. Matter 2011, 23, 213001.

(4) Ugeda, M. M.; et al. Characterization of collective ground states in single-layer $\mathrm{NbSe}_{2}$. Nat. Phys. 2016, 12, 92-97.

(5) Zhu, X.; Guo, J.; Zhang, J.; Plummer, E. W. Misconceptions associated with the origin of charge density waves. Adv. Phys. X 2017, 2, 622-640.

(6) Gabovich, A. M.; Voitenko, A. I.; Ausloos, M. Charge- and spindensity waves in existing superconductors: Competition between Cooper pairing and Peierls or excitonic instabilities. Phys. Rep. 2002, 367, 583-709.

(7) Chhowalla, M.; Shin, H.; Eda, G.; Li, L.; et al. The chemistry of two-dimensional layered transition metal dichalcogenide nanosheets. Nat. Chem. 2013, 5, 263.

(8) Manzeli, S.; Ovchinnikov, D.; Pasquier, D.; Yazyev, O. V.; Kis, A. 2D transition metal dichalcogenides. Nat. Rev. Mater. 2017, 2, 17033.

(9) Sugawara, K.; Nakata, Y.; Shimizu, R.; Han, P.; Hitosugi, T.; Sato, T.; Takahashi, T. Unconventional Charge-Density-Wave Transition in Monolayer 1T-TiSe 2 . ACS Nano 2016, 10, 1341-1345.

(10) Chen, P.; Pai, W. W.; Chan, Y. H.; Takayama, A.; Xu, C. Z.; Karn, A.; Hasegawa, S.; Chou, M. Y.; Mo, S. K.; Fedorov, A. V.; Chiang, T. C. Emergence of charge density waves and a pseudogap in single-layer $\mathrm{TiTe}_{2}$. Nat. Commun. 2017, 8, 516.

(11) Mulazzi, M.; Chainani, A.; Katayama, N.; Eguchi, R.; Matsunami, M.; Ohashi, H.; Senba, Y.; Nohara, M.; Uchida, M.; Takagi, H.; Shin, S. Absence of nesting in the charge-density-wave system $1 \mathrm{~T}-\mathrm{VS}_{2}$ as seen by photoelectron spectroscopy. Phys. Rev. B: Condens. Matter Mater. Phys. 2010, 82, 075130.

(12) Strocov, V. N.; Shi, M.; Kobayashi, M.; Monney, C.; Wang, X.; Krempasky, J.; Schmitt, T.; Patthey, L.; Berger, H.; Blaha, P. Threedimensional electron realm in $\mathrm{VSe}_{2}$ by soft-x-ray photoelectron spectroscopy: Origin of charge-density waves. Phys. Rev. Lett. 2012, 109, 086401.

(13) Arguello, C. J.; Chockalingam, S. P.; Rosenthal, E. P.; Zhao, L.; Gutiérrez, C.; Kang, J. H.; Chung, W. C.; Fernandes, R. M.; Jia, S.; Millis, A. J.; Cava, R. J.; Pasupathy, A. N. Visualizing the charge 
density wave transition in $2 \mathrm{H}-\mathrm{NbSe} 2$ in real space. Phys. Rev. B: Condens. Matter Mater. Phys. 2014, 89, 235115.

(14) Wilson, J. A.; di Salvo, F. J.; Mahajan, S. Charge-density waves and superlattices in the metallic layered transition metal dichalcogenides. Adv. Phys. 2001, 50, 1171-1248.

(15) Liu, R.; Tonjes, W. C.; Greanya, V. A.; Olson, C. G.; Frindt, R. F. Fermi surface of and its relation to the charge-density wave. Phys. Rev. B: Condens. Matter Mater. Phys. 2000, 61, 5212-5216.

(16) Enyashin, A. N.; Seifert, G. Density-functional study of $\mathrm{Li}_{x} \mathrm{MoS}_{2}$ intercalates $(0 \leq x \leq 1)$. Comput. Theor. Chem. 2012, 999, 13-20.

(17) Chen, X. B.; Chen, Z. L.; Li, J. Critical electronic structures controlling phase transitions induced by lithium ion intercalation in molybdenum disulphide. Chin. Sci. Bull. 2013, 58, 1632-1641.

(18) Rösner, M.; Haas, S.; Wehling, T. O. Phase diagram of electrondoped dichalcogenides. Phys. Rev. B: Condens. Matter Mater. Phys. 2014, 90, 245105.

(19) Zhuang, H. L.; Johannes, M. D.; Singh, A. K.; Hennig, R. G. Doping-controlled phase transitions in single-layer $\mathrm{MoS}_{2}$. Phys. Rev. B: Condens. Matter Mater. Phys. 2017, 96, 165305.

(20) Piatti, E.; Chen, Q.; Tortello, M.; Ye, J.; Gonnelli, R. S. Possible charge-density-wave signatures in the anomalous resistivity of $\mathrm{Li}$ intercalated multilayer MoS 2. Appl. Surf. Sci. 2018, 461, 269-275.

(21) Mak, K. F.; Lee, C.; Hone, J.; Shan, J.; Heinz, T. F. Atomically thin MoS2: A new direct-gap semiconductor. Phys. Rev. Lett. 2010, $105,136805$.

(22) Ye, J. T.; Zhang, Y. J.; Akashi, R.; Bahramy, M. S.; Arita, R.; Iwasa, Y. Superconducting dome in a gate-tuned band insulator. Science 2012, 338, 1193-1196.

(23) Zhang, R.; Tsai, I. L.; Chapman, J.; Khestanova, E.; Waters, J.; Grigorieva, I. V. Superconductivity in Potassium-Doped Metallic Polymorphs of $\mathrm{MoS}_{2}$. Nano Lett. 2016, 16, 629-636.

(24) Woollam, J. A.; Somoano, R. B. Superconducting critical fields of alkali and alkaline-earth intercalates of $\mathrm{MoS}_{2}$. Phys. Rev. B 1976, 13, 3843-3853.

(25) Lu, J. M.; Zheliuk, O.; Leermakers, I.; Yuan, N. F.; Zeitler, U.; Law, K. T.; Ye, J. T. Evidence for two-dimensional Ising superconductivity in gated $\mathrm{MoS}_{2}$. Science 2015, 350, 1353-1357.

(26) Kratochvilova, M.; Hillier, A. D.; Wildes, A. R.; Wang, L.; Cheong, S. W.; Park, J. G. The low-temperature highly correlated quantum phase in the charge-density-wave 1T-TaS2 compound. npj Quantum Mater. 2017, 2, 42.

(27) Splendiani, A.; Sun, L.; Zhang, Y.; Li, T.; Kim, J.; Chim, C. Y.; Galli, G.; Wang, F. Emerging photoluminescence in monolayer $\mathrm{MoS}_{2}$. Nano Lett. 2010, 10, 1271-1275.

(28) Andersen, A.; Kathmann, S. M.; Lilga, M. a.; Albrecht, K. O.; Hallen, R. T.; Mei, D. First-principles characterization of potassium intercalation in hexagonal $2 \mathrm{H}-\mathrm{MoS}_{2}$. J. Phys. Chem. C 2012, 116, $1826-1832$

(29) Sengoku, N.; Ogawa, K. Investigations of electronic structures of defects introduced by ar ion bombardments on $\mathrm{MoS}_{2}$ by scanning tunneling microscopy. Jpn. J. Appl. Phys. 1995, 34, 3363-3367.

(30) Bampoulis, P.; van Bremen, R.; Yao, Q.; Poelsema, B.; Zandvliet, H. J.; Sotthewes, K. Defect Dominated Charge Transport and Fermi Level Pinning in MoS2/Metal Contacts. ACS Appl. Mater. Interfaces 2017, 9, 19278-19286.

(31) Yan, S.; Iaia, D.; Morosan, E.; Fradkin, E.; Abbamonte, P.; Madhavan, V. Influence of Domain Walls in the Incommensurate Charge Density Wave State of Cu Intercalated 1T-TiSe2. Phys. Rev. Lett. 2017, 118, 106405.

(32) Chen, L.; Cheng, P.; Wu, K. Quasiparticle interference in unconventional 2D systems. J. Phys.: Condens. Matter 2017, 29, 103001.

(33) Soumyanarayanan, A.; Yee, M. M.; He, Y.; van Wezel, J.; Rahn, D. J.; Rossnagel, K.; Hudson, E. W.; Norman, M. R.; Hoffman, J. E. Quantum phase transition from triangular to stripe charge order in $\mathrm{NbSe}_{2}$. Proc. Natl. Acad. Sci. U. S. A. 2013, 110, 1623-1627.

(34) Novello, A. M.; Spera, M.; Scarfato, A.; Ubaldini, A.; Giannini, E.; Bowler, D. R.; Renner, C. Stripe and Short Range Order in the
Charge Density Wave of $1 \mathrm{~T}-\mathrm{Cu}_{x} \mathrm{TiSe}_{2}$. Phys. Rev. Lett. 2017, 118, 017002 .

(35) Eknapakul, T.; King, P. D. C.; Asakawa, M.; Buaphet, P.; He, R. H.; Mo, S. K.; Takagi, H.; Shen, K. M.; Baumberger, F.; Sasagawa, T.; Jungthawan, S.; Meevasana, W. Electronic structure of a quasifreestanding MoS2 monolayer. Nano Lett. 2014, 14, 1312-1316.

(36) Garcia-Goiricelaya, P.; Lafuente-Bartolome, J.; Gurtubay, I. G.; Eiguren, A. Emergence of large nonadiabatic effects induced by the electron-phonon interaction on the complex vibrational quasiparticle spectrum of doped monolayer $\mathrm{MoS}_{2}$. Phys. Rev. B: Condens. Matter Mater. Phys. 2020, 101, 54304.

(37) Gusakova, J.; Wang, X.; Shiau, L. L.; Krivosheeva, A.; Shaposhnikov, V.; Borisenko, V.; Gusakov, V.; Tay, B. K. Electronic Properties of Bulk and Monolayer TMDs: Theoretical Study Within DFT Framework (GVJ-2e Method). Phys. Status Solidi A 2017, 214, 1700218. 